\title{
Volume and topological invariants of quantum many-body systems
}

\author{
Xiao-Gang Wen ${ }^{1}$ and Zhenghan Wang ${ }^{2}$ \\ ${ }^{1}$ Department of Physics, Massachusetts Institute of Technology, Cambridge, Massachusetts 02139, USA \\ ${ }^{2}$ Microsoft Station $Q$ and Department of Mathematics, University of California, Santa Barbara, California 93106, USA
}

(Received 14 May 2019; revised 4 April 2020; accepted 8 May 2020; published 7 July 2020)

\begin{abstract}
Quantum many-body systems described by Lagrangian path integrals can realize many different topological phases of matter. One of the most important problems in condensed-matter physics is to extract topological invariants from the Lagrangian and to determine the topological order in the systems. In this paper, we suggest a general solution to this problem. Given a path integral on space-time lattice $C^{d+1}$ that describes a short-range correlated (i.e., gapped) system, we design systematic ways to extract topological invariants. For example, we show how to use nonuniversal partition functions $Z\left(C^{2+1}\right)$ on several space-time lattices with related topologies to extract $\left(M_{f}\right)_{11}$ and $\operatorname{Tr}\left(M_{f}\right)$, where $M_{f}$ is a representation of the modular group $\operatorname{SL}(2, \mathbf{Z})$, a topological invariant that characterizes $(2+1)$-dimensional topological orders. Our approach is guided by a notion of quantum volume. A path integral gives rise to a wave function $|\Psi\rangle$ on the boundary of $(d+1)$-dimensional space-time $C^{d+1}$. We show that $V=\ln \sqrt{\langle\Psi \mid \Psi\rangle}$ satisfies the inclusion-exclusion property $\frac{V(A \cup B)+V(A \cap B)}{V(A)+V(B)}=1$ and behaves like a volume of the space-time $C^{d+1}$ in the thermodynamics limit if the system is short range correlated. This leads to a proposal that the vector $|\Psi\rangle$ itself is the quantum volume of the space-time $C^{d+1}$. The subleading term of thermodynamics limit gives rise to the topological invariants.
\end{abstract}

DOI: 10.1103/PhysRevResearch.2.033030

\section{INTRODUCTION}

Recently, it was proposed that all force particles (the gauge bosons) and matter particles (the fermions) may arise from entangled quantum information if we assume the space to be an ocean of qubits [1-5]. If the physical space is, indeed, an entangled ocean of qubits, then it is natural to suspect that the mathematical notion of continuous space (i.e., the notion of a manifold) may also arise from entangled qubits that are discrete and algebraic in nature. This leads to the currently very active research direction of trying to view continuous geometry as emergent from discrete algebra. This point of view may lead to a quantum theory of gravity [6,7], a long-sought-after goal of fundamental theoretical physics. However, at the moment, we still do not know how the metrics of a manifold and an Einstein equation that governs the dynamics of metrics can emerge from discrete and entangled qubits if we require that the fluctuations of the metrics are the only low-energy excitations. (For the emergence of nonEinstein quantum gravity as the only low-energy dynamics, see Refs. [8-10].) In this paper, we will address a much simpler question: how the volume emerges from discrete and entangled qubits. We would like to demonstrate that at least one geometric quantity, the volume, can emerge from discrete algebra.

Published by the American Physical Society under the terms of the Creative Commons Attribution 4.0 International license. Further distribution of this work must maintain attribution to the author(s) and the published article's title, journal citation, and DOI.
It turns out that if we have only emergent volume, the associated space does not have a sense of "shape" and its dynamics is not governed by Einstein's theory of gravity, but by a different gravitational theory-a topological quantum field theory $[11,12]$ with an energy gap and short-range correlation. We may call this kind of gravity topological gravity. There are many examples to demonstrate how various topological gravities (i.e., various topological quantum field theories) emerge from entangled qubits (i.e., entangled manybody systems). The emergence of topological quantum field theories from entangled many-body systems is well studied in condensed-matter physics under the name of topological order $[13,14]$. Thus, entangled many-body systems can also give us topological gravity and a sense of volume: an emerging geometric property.

At first, the issue of emergent volume appears to be trivial for many-body systems since every many-body system has a natural definition of volume: the number of lattice sites. However, this works only for translation-symmetric manybody systems. For many-body systems without translation symmetry, it is not proper to define the volume as the number of lattice sites since different sites may have different contributions to the volume. Thus, we want to ask how to define the notion of volume for a non-translation-symmetric many-body systems on a lattice.

We find that if a quantum many-body system is in a topologically ordered phase (or more precisely, a gapped quantum liquid state $[15,16]$, not a foliated or fracton state [15,17-20]), then the notion of volume can be defined even without translation symmetry. Here, we stress that the above "volume" is not a property of the space-time complex $C_{N}^{d+1}$ but a property of space-time plus a many-body system defined 
on the space-time. A many-body system on the space-time can be defined via a tensor network characterized by a tensor $T$ (see Appendixes A and B for more details). Thus, the volume is a property of a pair $\left(C_{N}^{d+1}, T\right)$.

However, the volume that directly arises from the manybody system $T$ on $C_{N}^{d+1}$ is not exactly the volume in the familiar classical sense. We will call this notion of volume the quantum volume. Unlike the classical volume which is a positive real number, a quantum volume is not a real number, but a vector in a Hilbert space.

From the quantum volume of a many-body system, we may define an emergent classical volume as the norm of the quantum volume (i.e., the norm of the vector). We find that such a classical volume does not satisfy the classical-volume axioms exactly. However, in the large-system-size limit (i.e., when the number of vertices of space-time complex $C_{N}^{d+1}$ goes to infinity, $N \rightarrow \infty$ ), the leading term of the classical volume does satisfy the classical-volume axioms.

We also find that the finite subleading terms that violate the classical-volume axioms vanish for many-body states with trivial topological order (i.e., for product states). So nonvanishing subleading terms imply a nontrivial topological order. In fact, those finite subleading terms are topological invariants that characterize the underlying topological order. Extracting those topological invariants (i.e., the subleading terms) from the nonuniversal quantum volume of a manybody system in the $N \rightarrow \infty$ limit is the main topic of this paper.

This is very similar to entanglement entropy: the leading term of entanglement entropy can be used to define the total area of the interface, while the finite subleading term-the topological entanglement entropy-is a topological invariant that characterizes the underlying topological order [21,22]. We speculate that the two could be related by some generalization of Fubini's theorem.

We stress that the topological invariants discussed in this paper are properties of the pair $\left(C_{N}^{d+1}, T\right)$ : the space-time $C_{N}^{d+1}$ and the many-body system $T$. Thus, our topological invariants are not the usual topological invariants discussed in mathematics and topological quantum field theory (TQFT), but a stronger version of the usual topological invariants. The usual topological invariants are invariant under arbitrary homotopic deformation of space-time $C_{N}^{d+1}$. However, our topological invariants are invariant both under arbitrary homotopic deformation of space-time $C_{N}^{d+1}$ and under arbitrarily small deformations of the many-body system (i.e., under any small changes of the tensor $T$ ). Many topological invariants constructed from TQFT are not topological invariants in the above sense since those TQFT topological invariants are not invariant under some small changes of $T$ even in the $N \rightarrow$ $\infty$ limit. One main issue of this paper is to separate our strong topological invariants from those TQFT topological invariants. Only our strong topological invariants are universal properties of many-body systems in the thermodynamic limit. Only those strong topological invariants can be used to characterize different quantum phases (i.e., topological orders) in many-body systems. This observation may have important implications: the theories for topological phases of matter based on TQFT topological invariants may need to be reevaluated.

\section{VOLUME IN A QUANTUM MANY-BODY SYSTEM}

To define a many-body system through a space-time path integral, we first triangulate the $(d+1)$-dimensional spacetime to obtain a simplicial complex $C_{N}$ with $N$ vertices. The degrees of freedom of our lattice model live on the vertices (denoted by $v_{i}$, where $i$ labels the vertices), on the edges (denoted by $e_{i j}$, where $\langle i j\rangle$ labels the edges), etc. The action amplitude $e^{-S_{\text {cell }}}$ for an $n$ cell $(i j \cdots k)$ is a complex function of $v_{i}, e_{i j}, \ldots$ on the cell: $T_{i j \cdots k}\left(\left\{v_{i}\right\},\left\{e_{i j}\right\}, \ldots\right)$. The total action amplitude $e^{-S}$ for the configuration $\left\{v_{i}\right\},\left\{e_{i j}\right\}, \ldots$ (or a path) is given by

$$
e^{-S}=\prod_{(i j \cdots k)} T_{i j \cdots k}\left(\left\{v_{i}\right\},\left\{e_{i j}\right\}, \ldots\right),
$$

where $\prod_{(i j \cdots k)}$ is the product over all the $n$ cells $(i j \cdots k)$. Our lattice theory is defined by the following imaginary-time path integral (or partition function):

$$
Z\left(\left\{v_{i}^{\text {bdry }}, e_{i j}^{\text {bdry }}, \ldots\right\}, C_{N}^{d+1}, T\right)=\sum_{\left\{v_{i}\right\},\left\{e_{i j}\right\}, \ldots} e^{-S}:=\left|\Psi\left(C_{N}^{d+1}\right)\right\rangle,
$$

where $\sum_{\left\{v_{i}\right\},\left\{e_{i j}\right\}, \ldots}$ sums over only the indices inside the spacetime complex and the indices $v_{i}^{\text {bdry }}, e_{i j}^{\text {bdry }}, \ldots$ on the boundary of the space-time complex are fixed. We see that on the space-time complex with boundary, the path integral gives rise to a wave function $|\Psi\rangle$ on the boundary. On space-time complex with no boundary, the path integral gives rise to a complex number: the partition function $Z\left(C_{N}^{d+1}, T\right.$ ). (In the above discussion, some important details are ignored. A more precise description can be found in Appendixes A and B, as well as in Ref. [23].)

Using the wave function $|\Psi\rangle$, we would like to call $V\left(C_{N}^{d+1}, T\right)$, defined by

$$
V\left(C_{N}^{d+1}, T\right)=\ln \sqrt{\langle\Psi \mid \Psi\rangle},
$$

the (classical) space-time volume of the pair $\left(C_{N}^{d+1}, T\right)$. Such a definition is motivated by the following considerations. In the $N \rightarrow \infty$ thermodynamic limit, the partition function is roughly given by

$$
\begin{aligned}
& Z\left(\left\{v_{i}^{\text {bdry }}, e_{i j}^{\text {bdry }}\right\}, C_{N}^{d+1}, T\right) \\
& \quad=e^{-S_{N}^{\text {eff }}} Z^{\text {top }}\left(\left\{v_{i}^{\text {bdry }}, e_{i j}^{\text {bdry }}\right\}, C_{N}^{d+1}, T\right),
\end{aligned}
$$

where $S_{N}^{\text {eff }} \propto N$ is the integration of energy density over the space-time manifold and $Z^{\text {top }}\left(\left\{v_{i}^{\text {bdry }}, e_{i j}^{\text {bdry }}\right\}, C_{N}^{d+1}, T\right)$ is independent of $N$. (The notion of a topological partition function $Z^{\text {top }}$ and a topological path integral is discussed in more detail in Appendix and Ref. [23].) We see that the leading term $S_{N}^{\text {eff }}$ behaves like a volume, which leads our definition (2). In other words, the many-body system described by the tensor $T$ and triangulation $C_{N}^{d+1}$ gives rise to a classical volume of the space-time as in (2). In this paper, we assume energy density $<0$, so that $e^{-S_{N}^{\text {eff }}} \gg 1$, and the classical volume is positive. At the leading order of $N$, such a classical volume, $V\left(C_{N}^{d+1}, T\right)=-S_{N}^{\text {eff }}=-\int_{\text {space-time }}$ energy-density, satisfies the inclusion-exclusion property: Let $Y$ be a $d$-dimensional manifold with a Riemannian metric $\gamma$ and $\mathcal{M}_{(Y, \gamma)}$ be the set of all Riemannian manifolds $(X, g)$ such that $\partial X=Y$ and 
$\left.g\right|_{Y}=\gamma$. Then the volume functional $V: \mathcal{M}_{(Y, \gamma)} \rightarrow \mathbb{R}$ satisfies the inclusion-exclusion formula

$$
V(A)+V(B)=V(A \cup B)+V(A \cap B),
$$

where a space $M=A \cup B$ contains two overlapping regions $A$ and $B$, and the overlap is given by $A \cap B$.

We like to mention that the Euler characteristic $\chi(X)$ of a topological space $X$ usually appears in the path integrals as a prefactor $a^{\chi}$ (just like $e^{-S_{N}^{\text {eff }}}$ ) and behaves like a volume. The Euler characteristic has an axiomatic characterization. The Euler characteristic $\chi(X)$ is essentially the only homotopy invariant function on all topological spaces that satisfies the multiplicative property $\chi(X \times Y)=\chi(X) \chi(Y)$ and the inclusion-exclusion formula $\chi(X)=\chi(A)+\chi(B)-$ $\chi(A \cap B)$ if $X=A \cup B$. But there are no known axiomatic characterizations of the volume functional.

To discuss volume more precisely, i.e., to include both terms at the $N$ th order and the $N^{0}$ th order, it is better to introduce a notion of quantum volume, or q-volume. A q-volume is not a real number. It is a vector, i.e., the wave function $|\Psi\rangle$. The norm of the q-volume gives rise to the corresponding classical volume. We stress that the above definition of the q-volume is very general. It applies to both gapped many-body systems and gapless many-body systems. In this paper, we will concentrate on gapped many-body systems.

A topological invariant that is closely related to volume is the Gromov norm of the fundamental class of a manifold [24]. The Gromov norm behaves well with respect to covering maps, so one test for quantum volume would be to study its behavior under covering maps. Quantum volumes also satisfy some neutrality and gluing properties. For a manybody system described by tensor $T$, its q-volume satisfies

$$
\left|\Psi\left(C_{1}^{d+1} \cup C_{2}^{d+1}\right)\right\rangle=\sum_{\partial C_{1}^{d+1} \cap \partial C_{2}^{d+1}}\left[\left|\Psi\left(C_{1}^{d+1}\right)\right\rangle \otimes\left|\Psi\left(C_{2}^{d+1}\right)\right\rangle\right]
$$

if the space-time complexes $C_{1}^{d+1}$ and $C_{2}^{d+1}$ overlap only on their boundaries. We note that $\left|\Psi\left(C_{1}^{d+1}\right)\right\rangle$ and $\left|\Psi\left(C_{2}^{d+1}\right)\right\rangle$ are complex functions that depend on the indices on the boundaries $\partial C_{1}^{d+1}$ and $\partial C_{2}^{d+1}$. Here, $\sum_{\partial C_{1}^{d+1} \cap \partial C_{2}^{d+1}}$ sums over the indices inside the overlapped boundaries $\partial C_{1}^{d+1} \cap \partial C_{2}^{d+1}$ with a weight [for details see Eq. (C1)]. Since $|\Psi\rangle$ 's are exponentially large $e^{-S_{N}^{\text {eff }}}$ in the large- $N$ limit, from Eq. (5), we can show that, in the thermodynamic limit, the corresponding classical volume satisfies $\frac{V\left(C_{1}^{d+1} \cup C_{2}^{d+1}\right)}{V\left(C_{1}^{d+1}\right)+V\left(C_{2}^{d+1}\right)}=1$, which is a special case of Eq. (4).

\section{TOPOLOGICAL INVARIANT THROUGH Q-VOLUME AND SURGERY}

In general the partition function $Z\left(C_{N}^{d+1}, T\right)=e^{-S_{N}^{\text {eff }}} Z^{\text {top }}$ of a many-body system is not a topological invariant even when the many-body system described by the tensor $T$ realizes a topologically ordered state. In the absence of translation symmetry, it is not trivial to separate the nonuniversal part $e^{-S_{N}^{\text {eff }}}$ from the topological invariant $Z^{\text {top }}$ by just knowing $Z\left(C_{N}^{d+1}, T\right)$.

To achieve the separation, we note that the $S_{N}^{\text {eff }}$ part of $\ln \left|Z\left(C_{N}^{d+1}, T\right)\right|$ is the standard classical volume of the space- time that satisfies the inclusion-exclusion property (4). Thus, we can separate the $Z^{\text {top }}$ part of the partition function since $Z^{\text {top }}$ violates the properties of classical volume. $Z^{\text {top }}$ is the topological invariant that reflects the nontrivial topological order in the system. In other words, for a system with trivial topological order, the classical volume axioms are satisfied even at $e^{N^{0}}$ order, i.e., $Z^{\text {top }}=1$ [see Eq. (7)].

As an application of the above idea, we choose a special pair of triangulated closed space-time complexes $M_{1}$ and $M_{2}$ (in the fine-triangulation limit) and consider the following ratio of two partition functions for a many-body system described by a tensor $T$ :

$$
Z\left(M_{1}, T\right) / Z\left(M_{2}, T\right)
$$

To choose proper $M_{1}$ and $M_{2}$, we may start with a complex $\tilde{M}$ with $2 n$ disconnected but isomorphic boundaries. $M_{1}$ and $M_{2}$ are formed by gluing those boundaries together in different ways. For example, we may choose $\tilde{M}=M_{U} \sqcup M_{D} \sqcup N_{U} \sqcup$ $N_{D}$, where the boundaries of $M_{U}, M_{D}, N_{U}$, and $N_{D}$ are complexes all isomorphic to $B$. Gluing the boundaries in different

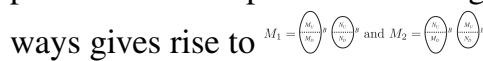

If $V=\ln |Z|$ exactly satisfies the inclusion-exclusion property of the classical volume, then the above ratio (6) will be 1 . However, in general, the subleading $N^{0}$ term in $V=$ $\ln |Z|$ does not satisfy the inclusion-exclusion property. Such subleading terms will make the ratio (6) differ from 1 . But for a system $T$ with trivial topological order, we find that the above ratio (6) will be 1 in the thermodynamic limit. This is because the partition functions and their ratio are invariant under the tensor network renormalization transformations which coarse grain the tensor network away from the boundary $B$. If the tensor $T$ describes a trivial topological order, the tensor network will flow to a corner-double-line tensor network in $1+1$ dimension or a similar structured tensor network in higher dimensions [25]:

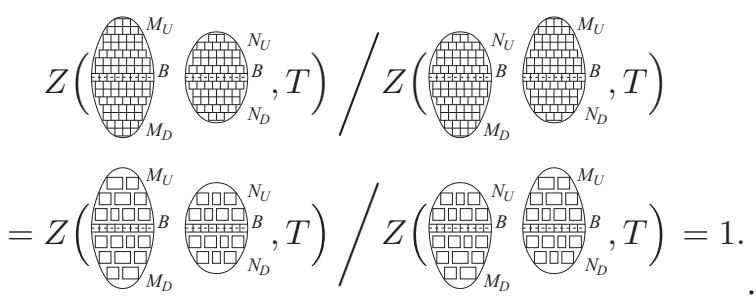

This allows us to show the ratio (6) is 1 if the system has no topological order. Thus, the ratio (6) is a topological invariant that can characterize nontrivial topological orders in the system.

We may also choose $\bar{M}=I \times B \sqcup I \times B$, where $B$ is a closed complex corresponding to the triangulated space. Such an $M$ has four disconnected boundaries, all isomorphic to $B$. Gluing the boundaries in different ways gives rise to $M_{1}$ and $M_{2}$ :

$$
M_{1}={ }^{B} \bigcirc{ }_{I \times B}{ }_{B}, \quad M_{2}={ }_{I \times B} \overbrace{I \times B} \bigodot^{I \times B} .
$$


In fact, for such a choice, the corresponding ratio gives rise to the ground-state degeneracy (GSD) on space $B$ :

$$
G S D(B)=Z\left({ }^{B} \bigodot_{I \times B}^{\bigcirc}{ }^{B} \bigodot_{I \times B}^{\bigcirc} ; T\right) / Z\left({ }_{I \times B} \bigodot^{B} ; T\right) .
$$

The following ratio is also a topological invariant:

$$
Z\left(\left(\begin{array}{c}
M_{U} \\
M_{D}
\end{array}\right)^{B}, T\right) / \sqrt{\left.Z\left(\left(\begin{array}{c}
M_{U} \\
\bar{M}_{U}
\end{array}\right)^{B}, T\right) Z\left(\bar{M}_{D}\right)^{B}, T\right)}
$$

The above ratio is calculated by dividing the closed space-time $M$ into two parts, $M=M_{U} \cup M_{D}$. It not only dependent on the space-time $M$, but it also depends on $M_{U}$ and $M_{D}$, i.e., how we partition $M$. Notice that the space-times with boundaries, $M_{U}$ and $M_{D}$, give rise to two vectors, $\left\langle\Psi\left(M_{U}\right)\right|$ and $\left|\Psi\left(M_{D}\right)\right\rangle$, which are not normalized. The above ratio is simply the overlap of $\left\langle\Psi\left(M_{U}\right)\right|$ and $\left|\Psi\left(M_{D}\right)\right\rangle$ after normalization: $\left\langle\Psi\left(M_{U}\right) \mid \Psi\left(M_{D}\right)\right\rangle$

$\sqrt{\left\langle\Psi\left(M_{U}\right) \mid \Psi\left(M_{U}\right)\right\rangle} \sqrt{\left\langle\Psi\left(M_{D}\right) \mid \Psi\left(M_{D}\right)\right\rangle} \cdot$

Let us apply the above approaches to construct some topological invariants. First, for $(d+1)$-dimensional $[(d+1) \mathrm{D}]$ many-body systems with a unique gapped liquid ground state on $S^{d}$, we have the following result $[11,26]$ :

$$
\begin{aligned}
& \left.\left.Z\left(\begin{array}{c}
M_{U} \\
\hdashline M_{D}
\end{array}\right) B\left(\begin{array}{c}
N_{U} \\
N_{D}
\end{array}\right)^{B}, T\right) / Z\left(\begin{array}{c}
N_{U} \\
M_{D}
\end{array}\right) B\left(\begin{array}{c}
M_{U} \\
N_{D}
\end{array}\right)^{B}, T\right) \\
= & \frac{Z\left(M_{U} \cup M_{D}\right) Z\left(N_{U} \cup N_{D}\right)}{Z\left(N_{U} \cup M_{D}\right) Z\left(M_{U} \cup N_{D}\right)}=\left.\right|_{B=S^{d}} 1
\end{aligned}
$$

So when the partition boundary is a sphere $B=S^{d}$, the above ratio fails to give rise to any nontrivial topological invariant. Thus, the connected sum decomposition does not give rise to nontrivial topological invariants. The nontrivial topological invariants may arise when the division has a nontrivial cross section $B$ beyond a sphere.

One such topological invariant is obtained by choosing $M_{U}=D^{2} \times S^{1}, M_{D}=D^{2} \times S^{1}, N_{U}=S^{1} \times D^{2}, N_{D}=S^{1} \times$ $D^{2}$. We find

$$
\begin{gathered}
\frac{Z\left(D^{2} \times S^{1} \cup D^{2} \times S^{1}\right) Z\left(S^{1} \times D^{2} \cup S^{1} \times D^{2}\right)}{Z\left(S^{1} \times D^{2} \cup D^{2} \times S^{1}\right) Z\left(D^{2} \times S^{1} \cup S^{1} \times D^{2}\right)} \\
=\left.\right|_{N \rightarrow \infty}\left(\frac{Z^{\text {top }}\left(S^{2} \times S^{1}\right)}{Z^{\text {top }}\left(S^{3}\right)}\right)^{2}=D^{2},
\end{gathered}
$$

which allows us to calculate the total quantum dimension $D^{2}=\sum d_{i}^{2}$ of a $(2+1) \mathrm{D}$ topologically ordered state. Here, $D^{2} \times S^{1} \cup D^{2} \times S^{1}=S^{2} \times S^{1}$ is obtained by gluing two solid tori $D^{2} \times S^{1}$, and $S^{1} \times D^{2} \cup D^{2} \times S^{1}=S^{3}$ is obtained by gluing two solid tori in a twisted way.

Also $N \rightarrow \infty$ is the limit of more and more refined triangulation of the space-time (i.e., the thermodynamics limit in condensed-matter physics). To obtain the first equal sign in Eq. (12) we have used the fact that the leading term $S_{N}^{\text {eff }}$ in the partition function is given by the integration of local energy density over space-time. Thus, the leading $S_{N}^{\text {eff }}$ satisfies the inclusion-exclusion property of the classical volume in the $N \rightarrow \infty$ limit, and their contributions are canceled in the ratio. This leads to the first equal sign in Eq. (12).

Another topological invariant is given by

$$
\begin{aligned}
& \frac{Z\left(D^{2} \times S^{1} \times S^{1} \cup D^{2} \times S^{1} \times S^{1}\right)}{Z\left(S^{1} \times D^{2} \times S^{1} \cup D^{2} \times S^{1} \times S^{1}\right)} \\
& \quad \times \frac{Z\left(S^{1} \times D^{2} \times S^{1} \cup S^{1} \times D^{2} \times S^{1}\right)}{Z\left(D^{2} \times S^{1} \times S^{1} \cup S^{1} \times D^{2} \times S^{1}\right)} \\
& =\left.\right|_{N \rightarrow \infty}\left(\frac{Z^{\text {top }}\left(S^{2} \times S^{1} \times S^{1}\right)}{Z^{\text {top }}\left(S^{3} \times S^{1}\right)}\right)^{2}=N_{p}^{2},
\end{aligned}
$$

which allows us to calculate the number $N_{p}$ of topological types of pointlike excitations of a $(3+1) \mathrm{D}$ topologically ordered state.

We can also use Eq. (10) to construct more topological invariants. First, let $M_{U}$ and $M_{D}$ be handlebodies of genus $g$, and let $f$ be an orientation reversing homeomorphism from the boundary of $B=\partial M_{U}$ to the boundary of $B=\partial M_{D}$. By gluing $M_{U}$ to $M_{D}$ along $B$ we obtain a compact oriented three-manifold $M=V \cup_{f} W$. Every closed, orientable threemanifold may be so obtained, which is called a Heegaard splitting. Thus, we can construct a topological invariant for each orientable three-manifold and its Heegaard splitting.

More specifically, we can choose $M_{U}=D^{2} \times S^{1}$ and $M_{D}=D^{2} \times S^{1}$ and let $f$ be a mapping from $S^{1} \times S^{1}$ to $S^{1} \times S^{1}$. Thus, $f$ is an element in $\operatorname{SL}(2, \mathbb{Z})$. In this case we find that

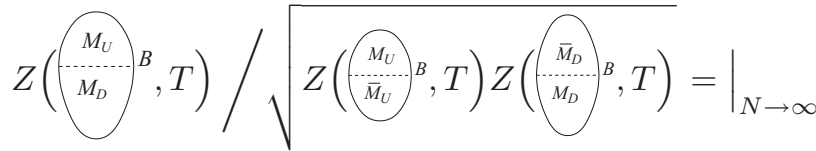

$$
\begin{aligned}
& Z^{\operatorname{top}}\left({\stackrel{M}{M_{U}}}_{M_{D}}^{B}, T\right) / \sqrt{\left.\left.Z^{\operatorname{top}(}\left(\begin{array}{c}
M_{U} \\
\bar{M}_{U}
\end{array}\right)^{B}, T\right) Z^{\operatorname{top}(}\left(\begin{array}{c}
\bar{M}_{D} \\
M_{D}
\end{array}\right)^{B}, T\right)} \\
& =\left(M_{f}\right)_{11} \text {, }
\end{aligned}
$$

where $M_{f}$ is the representation of $\operatorname{SL}(2, \mathbb{Z})$ in the quasiparticle basis $[13,27,28]$.

We may also choose $M_{U}=I^{1} \times T^{2} \cup_{f} I^{1} \times T^{2}$ and $M_{D}=$ $I^{1} \times T^{2}$. Note that $I^{1} \times T^{2}$ has two $T^{2}=S^{1} \times S^{1}$ boundaries. $M_{U}$ is formed by two $I^{1} \times T^{2}$ 's glued along one of the $T^{2}$ boundaries with an $f$ twist. Then, we glue the two $T^{2}$ boundaries of $M_{U}$ and two $T^{2}$ boundaries of $M_{D}$ directly without twist to form the total space-time lattice. In this case we find that

$$
\begin{aligned}
& Z\left(\left(\begin{array}{c}
M_{U} \\
M_{D}
\end{array}\right)^{B}, T\right) / \sqrt{\left.Z\left(\left(\begin{array}{c}
M_{U} \\
\bar{M}_{U}
\end{array}\right)^{B}, T\right) Z\left({\overline{M_{D}}}_{M_{D}}\right)^{B}, T\right)}=\left.\right|_{N \rightarrow \infty}
\end{aligned}
$$

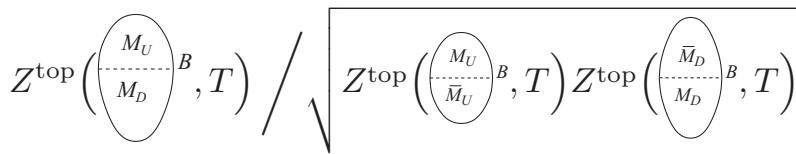

$$
\begin{aligned}
& =\operatorname{Tr}\left(M_{f}\right) / \operatorname{dim} M_{f} \text {. }
\end{aligned}
$$


$M_{f}$ is an important topological invariant that characterizes the topological order in the many-body system.

The above expression allows us to compute the topological invariants $\left(M_{f}\right)_{11}$ and $\operatorname{Tr}\left(M_{f}\right)$ using a generic nonfixed-point path integral ${ }^{8}$ in the thermodynamical limit. We stress that in Eq. (14), we need to choose the triangulation on $M_{U}=D^{2} \times$ $S^{1}$ and $M_{D}=D^{2} \times S^{1}$, such that the induced triangulation on the common boundary $B=\partial D^{2} \times S^{1}$ is related by the mapping $f$.

We know that $\operatorname{SL}(2, \mathbb{Z})$ is generated by $S$ for $f=\left(\begin{array}{cc}0 & -1 \\ 1 & 0\end{array}\right)$ and $T$ for $f=\left(\begin{array}{ll}1 & 1 \\ 0 & 1\end{array}\right)$. By choosing different $f$ 's, we can obtain $S_{11}, T_{11}$, $(S T)_{11}$, etc., in the quasiparticles basis.

The last topological invariant (15) can be generalized easily to topological order in any dimension by replacing the torus $T^{2}$ by any closed orientable manifold $M^{d}$. This will give rise to a topological invariant $\operatorname{Tr}\left(M_{f}\right) / \operatorname{dim} M_{f}$, where $M_{f}$ is a representation of the mapping class group formed by $f$ : $M^{d} \rightarrow M^{d}$.

\section{SUMMARY}

We introduced the notion of quantum volume for quantum many-body systems defined on space-time lattice. The quantum volume is not a positive number but a vector in a Hilbert space which satisfies an additive property (5). We showed that the norm of the quantum volume gives rise to the classical volume that satisfies the inclusion-exclusion property (4) in the thermodynamic limit.

For a many-body system with topological order, its partition function is not universal and depends on the details of interaction. Using the idea of quantum volume and classical volume, we showed how to compute topological invariants from nonuniversal partition functions. In particular, we showed how to compute the trace and the $(1,1)$ matrix element of the modular representation in quasiparticle basis from nonuniversal $(2+1) \mathrm{D}$ partition functions.

\section{ACKNOWLEDGMENTS}

X.-G.W. is partially supported by NSF under Grants No. DMR-1506475 and No. DMS-1664412. Z.W. is partially funded by NSF under Grants No. DMS-1411212 and No. FRG-1664351.

\section{APPENDIX A: MANY-BODY SYSTEMS AND THE PATH INTEGRAL ON A SPACE-TIME LATTICE}

In this Appendix, we will define many-body systems without translation symmetry via the space-time path integral. We will define the space-time path integral using uniform tensors on an arbitrary random space-time lattice. Although the tensors are uniform, the random space-time lattice breaks the translation symmetry. Later, we will use such a space-time path integral to define the quantum volume of the random space-time lattice.

\section{Space-time complex}

To define a many-body system through a space-time path integral, we first triangulate the $n$-dimensional space-time to obtain a simplicial complex $C_{N}$ (see Fig. 1). Here, we assume

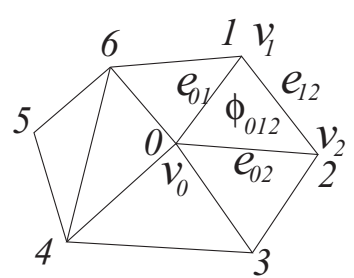

FIG. 1. A two-dimensional complex. The vertices (zerosimplices) are labeled by $i$. The edges (one-simplices) are labeled by $\langle i j\rangle$. The faces (two-simplices) are labeled by $\langle i j k\rangle$. The degrees of freedom may live on the vertices (labeled by $v_{i}$ ), on the edges (labeled by $e_{i j}$ ), and on the faces (labeled by $\phi_{i j k}$ ).

that all simplicial complexes are of bounded geometry in the sense that the number of edges that connect to one vertex is bounded by a fixed value. Also, the number of triangles that connect to one edge is bounded by a fixed value and so on.

In order to define a generic lattice theory on the space-time complex $C_{N}$, it is important to give the vertices of each simplex a local order. A nice local scheme to order the vertices is given by a branching structure [29-31]. A branching structure is a choice of orientation of each edge in the $n$-dimensional complex so that there is no oriented loop on any triangle (see Fig. 2).

The branching structure induces a local order of the vertices on each simplex. The first vertex of a simplex is the vertex with no incoming edges, and the second vertex is the vertex with only one incoming edge and so on. So the simplex in Fig. 2(a) has the following vertex ordering: $0<1<2<3$.

The branching structure also gives the simplex (and its subsimplexes) an orientation denoted by $s_{i j \cdots k}=1, *$. Figure 2 illustrates two three-simplices with opposite orientations $s_{0123}=1$ and $s_{0123}=*$. The red arrows indicate the orientations of the two-simplices which are the subsimplices of the three-simplices. The black arrows on the edges indicate the orientations of the one-simplices.

\section{Path integral on a space-time complex}

The degrees of freedom of our lattice model live on the vertices (denoted by $v_{i}$, where $i$ labels the vertices), on the edges (denoted by $e_{i j}$, where $\langle i j\rangle$ labels the edges), and on other high-dimensional simplicies of the space-time complex (see Fig. 1). The action amplitude $e^{-S_{\text {cell }}}$ for an $n$ cell $(i j \cdots k)$ is a complex function of $v_{i}, e_{i j}, \cdots: T_{i j \cdots k}\left(\left\{v_{i}\right\},\left\{e_{i j}\right\}, \ldots\right)$. The total action amplitude $e^{-S}$ for a configuration $\left\{v_{i}\right\},\left\{e_{i j}\right\}, \ldots$
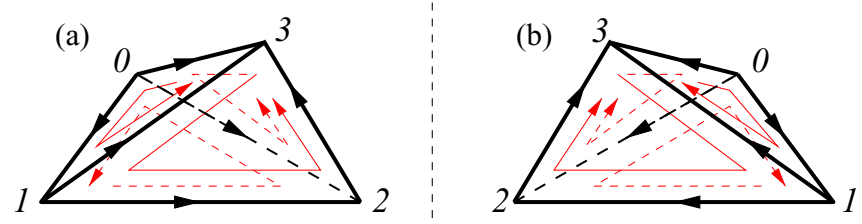

FIG. 2. Two branched simplices with opposite orientations. (a) A branched simplex with positive orientation and (b) a branched simplex with negative orientation. 
(or a path) is given by

$$
e^{-S}=\prod_{(i j \cdots k)}\left[T_{i j \cdots k}\left(\left\{v_{i}\right\},\left\{e_{i j}\right\}, \ldots\right)\right]^{s_{i j \cdots k}},
$$

where $\prod_{(i j \cdots k)}$ is the product over all the $n$ cells $(i j \cdots k)$. Note that the contribution from an $n$ cell $(i j \cdots k)$ is $T_{i j \cdots k}\left(\left\{v_{i}\right\},\left\{e_{i j}\right\}, \ldots\right)$ or $T_{i j \cdots k}^{*}\left(\left\{v_{i}\right\},\left\{e_{i j}\right\}, \ldots\right)$ depending on the orientation $s_{i j \cdots k}$ of the cell. Our lattice theory is defined by the following imaginary-time path integral (or partition function):

$$
Z=\sum_{\left\{v_{i}\right\},\left\{e_{i j}\right\}, \ldots} \prod_{(i j \cdots k)}\left[T_{i j \cdots k}\left(\left\{v_{i}\right\},\left\{e_{i j}\right\}, \ldots\right)\right]^{s_{i j \cdots k}} .
$$

Clearly, the partition function $Z$ depends on the space-time $M$, so we denote it as $Z(M)$. It is also clear that the partition function on a disjoint union of $M$ and $N$ is given by the product of the two partition functions on $M$ and on $N$ :

$$
Z(M \sqcup N)=Z(M) Z(N) .
$$

We would like to point out that, in general, the path integral may also depend on some additional weighting factors $w_{v_{i}}$, $d_{e_{i j}}^{v_{i} v_{j}}$, etc. [see Eq. (B3)]. In this section, for simplicity, we will assume those weighting factors are all equal to 1 .

In path integral (A2), we have assigned the same action amplitude $T_{i j \cdots k}\left(\left\{v_{i}\right\},\left\{e_{i j}\right\}, \ldots\right)$ to each simplex $(i j \cdots k)$. Such a path integral is called a uniform path integral. For simplicity, in this paper, we study only systems described by a uniform path integral. But our discussion also applies the more complicated cases where different simplices $(i j \cdots k)$ have different action amplitudes.

\section{Path integral on a space-time complex with a boundary}

In the last section, we defined the path integral on a space-time complex without a boundary. In this case, all the indices on vertices, edges, etc., are summed over. The resulting partition function $Z$ is just a complex number.

If the space-time manifold has a boundary $\partial M^{d+1}=M_{B}^{d}$, then the triangulation $C_{N}^{d+1}$ of $M^{d+1}$ has the following property: all the vertices in $C_{N}^{d+1}$ that are on the boundary $M_{B}^{d}$ form a subcomplex $B_{N^{\prime}}^{d}$, such that $B_{N^{\prime}}^{d}$ is a triangulation of $M_{B}^{d}$. In this case, we say that the complex $C_{N}^{d+1}$ has a boundary which is given by $B_{N^{\prime}}^{d} \equiv \partial C_{N}^{d+1}$.

The path integral on $C_{N}^{d+1}$ with a boundary is defined differently: we sum over only the indices on vertices, edges, etc., that are not on the boundary $B_{N^{\prime}}^{d}$. The indices on the boundary $B_{N^{\prime}}^{d}$ are fixed. So the resulting partition function $Z$ is a function of the indices on the boundary $B_{N^{\prime}}^{d}$. We see that the boundary $B_{N^{\prime}}^{d}$ gives rise to a Hilbert space $\mathcal{H}_{B_{N^{\prime}}^{d}}$ formed by all the complex functions of the indices on the boundary $B_{N^{\prime}}^{d}$. The partition function $Z$ on $C_{N}^{d+1}$ is a vector in $\mathcal{H}_{B_{N^{\prime}}^{d}}$ (i.e., a particular complex function of the indices on $B_{N^{\prime}}^{d}$ ). This is consistent with Atiyah's definition of topological quantum field theory [12].

\section{Path integral and Hamiltonian}

Consider a space-time complex of topology $M_{\text {space }} \times I$, where $I=\left[t, t^{\prime}\right]$ represents the time dimension and $M_{\text {space }}$ is

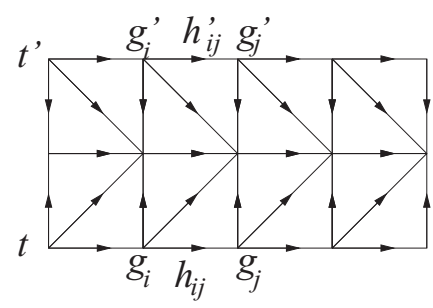

FIG. 3. Each time step of evolution is given by the path integral on a particular form of branched graph. Here is an example in $(1+1) \mathrm{D}$.

a closed space complex (see Fig. 3). The space-time complex $M_{\text {space }} \times I$ has two boundaries: one at time $t$ and another at time $t^{\prime}$. A path integral on the space-time complex $M_{\text {space }} \times I$ is a function of the indices on the two boundaries, which give us an amplitude $Z\left[\left\{v_{i}^{\prime}, e_{i j}^{\prime}, \ldots\right\},\left\{v_{i}, e_{i j}, \ldots\right\}\right]$ from a configuration $\left\{v_{i}, e_{i j}, \ldots\right\}$ at $t$ to another configuration $\left\{v_{i}^{\prime}, e_{i j}^{\prime}, \ldots\right\}$ at $t^{\prime}$. Here, $\left\{v_{i}, e_{i j}, \ldots\right\}$ and $\left\{v_{i}^{\prime}, e_{i j}^{\prime}, \ldots\right\}$ are the degrees of freedom on the boundaries (see Fig. 3 and Fig. 4). We interpret $Z\left[\left\{v_{i}^{\prime}, e_{i j}^{\prime}, \ldots\right\},\left\{v_{i}, e_{i j}, \ldots\right\}\right]$ as the amplitude of an evolution in imaginary time by a Hamiltonian:

$$
\begin{aligned}
& Z\left[\left\{v_{i}^{\prime}, e_{i j}^{\prime}, \ldots\right\},\left\{v_{i}, e_{i j}, \ldots\right\}\right] \\
& \quad=\left\langle v_{i}^{\prime}, e_{i j}^{\prime}, \ldots\left|\mathrm{e}^{-\left(t^{\prime}-t\right) H}\right| v_{i}, e_{i j}, \ldots\right\rangle .
\end{aligned}
$$

However, such an interpretation may not be valid since $Z\left[\left\{v_{i}^{\prime}, e_{i j}^{\prime}, \ldots\right\},\left\{v_{i}, e_{i j}, \ldots\right\}\right]$ may not give raise to a Hermitian matrix. It is a worrisome realization that the path integral and Hamiltonian evolution may not be directly related.

Here, we would like to use the fact that the path integrals that we are considering are defined on branched graphs with a "reflection" property [see Eq. (A1)]. We would like to show that such a path integral is related to a Hamiltonian evolution. The key is to require that each time step of evolution is given by branched graphs of the form in Fig. 3. One can show that $Z\left[\left\{v_{i}^{\prime}, e_{i j}^{\prime}, \ldots\right\},\left\{v_{i}, e_{i j}, \ldots\right\}\right]$, obtained by summing over all in the internal indices in the branched graphs (Fig. 3), has the form

$$
\begin{aligned}
& Z\left[\left\{v_{i}^{\prime}, e_{i j}^{\prime}, \ldots\right\},\left\{v_{i}, e_{i j}, \ldots\right\}\right] \\
& =\sum_{\left\{v_{i}^{\prime \prime}, e_{i j}^{\prime \prime}, \ldots\right\}} U^{*}\left[\left\{v_{i}^{\prime \prime}, e_{i j}^{\prime \prime}, \ldots\right\},\left\{v_{i}^{\prime}, e_{i j}^{\prime}, \ldots\right\}\right] \\
& \quad \times U\left[\left\{v_{i}^{\prime \prime}, e_{i j}^{\prime \prime}, \ldots\right\},\left\{v_{i}, e_{i j}, \ldots\right\}\right]
\end{aligned}
$$

and represents a positive-definite Hermitian matrix. Thus, a path integral of the form of Eq. (A1) always corresponds to

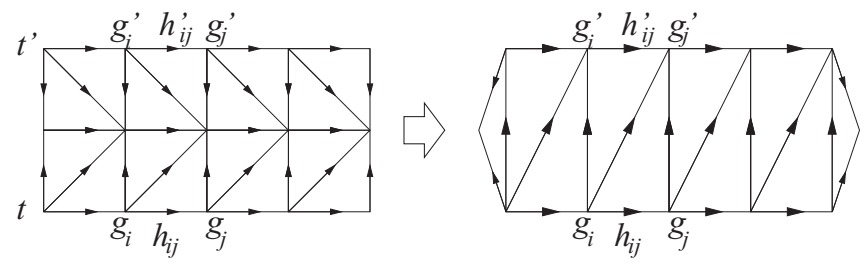

FIG. 4. The reduction of the double-layer time step to a singlelayer time step in space with a boundary for a $(1+1) \mathrm{D}$ topological path integral. 
a Hamiltonian evolution in imaginary time. In fact, the above $Z\left[\left\{v_{i}^{\prime}, e_{i j}^{\prime}, \ldots\right\},\left\{v_{i}, e_{i j}, \ldots\right\}\right]$ can be viewed as an imaginarytime evolution $T=e^{-\Delta \tau H}$ for a single time step.

\section{APPENDIX B: TOPOLOGICAL PATH INTEGRAL}

In this Appendix, we will review some results from Ref. [23].

\section{Topological path integral and topological orders with a gappable boundary}

The ground states of some many-body systems can have a special property that the ground states for systems with different sizes have only a different stacking of the product state, up to a local unitary transformation:

$$
\left|\Psi_{N_{2}}\right\rangle=U_{L U}\left|\Psi_{N_{1}}\right\rangle \otimes\left|\Psi_{P S}\right\rangle,
$$

where $N_{2}>N_{1}$ describes the system size, $\left|\Psi_{P S}\right\rangle$ is a product state for a system of size $N_{2}-N_{1}$, and $U_{L U}$ is a local unitary transformation. Such ground states are called gapped liquid states $[15,16]$. The gapped liquid states formally define the topologically ordered states $[13,14]$.

As many-body systems, all topologically ordered states are described by path integrals, and a path integral can be described by a tensor network (TN) with finite-dimensional tensors on a space-time lattice (i.e., a space-time complex). Even though topologically ordered states are all gapped, only some of them can be described by the so-called fixed-point path integrals, which are called topological path integrals.

Definition 1. Topological path integral. (1) A topological path integral has an action amplitude that can be described by a TN with finite-dimensional tensors. (2) It is a sum of the action amplitudes for all the paths. (The summation corresponds to the tensor contraction.) (3) Such a sum [called the partition function $Z^{\text {top }}(M)$ ] on a closed space-time $M$ depends on only the topology of the space-time. The partition function is invariant under the local deformations and reconnections of the TN.

In the next section, we will give concrete examples of the topological path integrals. The topological path integrals are closely related to topological orders with gappable boundary [32-35]. We conjecture the following [23]:

Conjecture 1. All topological orders with a gapped boundary are described by topological path integrals.

We make such a conjecture because we believe that the tensor network representation that we are going to discuss is the most general one. It can capture all possible fixedpoint tensors [36] under renormalization flow generated by the coarse graining of the TN [37,38], and those fixed-point tensors give rise to topological path integrals.

We also remark that we cannot say that all topological path integrals describe topological orders with a gapped boundary since some topological path integrals are stable while others are unstable (which means a small perturbation of the tensors will result in a different fixed-point tensor under renormalization flow). Only the stable topological path integrals describe topological order. Here we conjecture the following [23]:

Conjecture 2. A topological path integral in $(d+1)$ dimensional space-time constructed with finite-dimensional tensors is stable iff the partition function of the topological path integral satisfies $\left|Z^{\text {top }}\left(S^{1} \times S^{d}\right)\right|=1$.

Note that $Z^{\text {top }}\left(S^{1} \times S^{d}\right)$ is the ground-state degeneracy on $d$-dimensional space $S^{d}$. If a system has a gap and the ground degeneracy is 1 , a small perturbation cannot do much to destabilize the state. So $Z^{\text {top }}\left(S^{1} \times S^{d}\right)=1$ is a sufficient condition for a stable topological path integral. This argument implies that if the ground degeneracy is 1 on $S^{d}$, then the system has no locally distinguishable ground state, and the ground-state degeneracies in the spaces with other topologies are all robust against any small perturbation.

Since the topological path integrals are independent of retriangulation of the space-time, the partition function on a closed space-time depends on only the topology of the space-time. We point out that two topological path integrals, $Z^{\text {top }}(M)$ and $\tilde{Z}^{\text {top }}(M)$, can be smoothly connected if the two topological path integrals differ by

$$
Z^{\mathrm{top}}(M) / \tilde{Z}^{\mathrm{top}}(M)=W^{\chi(M)} e^{i \sum_{\left\{n_{i}\right\}} \phi_{n_{1} n_{2} \ldots} \int_{M} P_{n_{1} n_{2} \cdots}}
$$

where $\chi(M)$ is the Euler number of $M$ and $P_{n_{1} n_{2} \ldots}$ are combinations of Pontryagin classes: $P_{n_{1} n_{2} \cdots}=p_{n_{1}} \wedge p_{n_{2}} \wedge \cdots$ on $M$. $Z^{\text {top }}(M)$ and $\tilde{Z}^{\text {top }}(M)$ are connected since complex numbers $W$ and $\phi_{n_{1} n_{2} \ldots}$ are not quantized.

Equation (B2) may be the only local topological invariant that is not quantized (i.e., $W$ and $\phi_{n_{1} n_{2} \ldots}$ can be any complex numbers). Thus, we have the following conjecture [23]:

Conjecture 3. $Z^{\text {top }}(M)$ and $\tilde{Z}^{\text {top }}(M)$ are connected iff they are related by $\mathrm{Eq}$. (B2).

In other words, if two topological path integrals produce two topology-dependent partition functions that differ by a factor $W^{\chi(M)} e^{i \sum_{\left\{n_{i}\right\}} \phi_{n_{1} n_{2} \cdots} \int_{M} P_{n_{1} n_{2} \cdots} \text {, then the two topological path }}$ integrals describe the same topological order.

The above discussion is summarized as follows:

(1) All topological orders with a gappable boundary are described by a stable topological path integral constructed with finite-dimensional tensors.

(2) All stable topological path integrals describe topological orders with a gappable boundary.

(3) All stable topological path integrals related by Eq. (B2) describe the same topological order.

So the stable topological path integrals lead to a classification of topological orders with a gappable boundary.

\section{Examples of topological path integrals in $(2+1) D$}

The topological path integral that describes a $(2+1) \mathrm{D}$ topologically ordered state with a gapped boundary can be constructed from a tensor set $T$ of two real and one complex tensors: $T=\left(w_{v_{0}}, d_{e_{01}}^{v_{0} v_{1}}, C_{v_{0} v_{1} v_{2} v_{3} ; \phi_{013} \phi_{123}}^{e_{01} e_{22} e_{03} e_{12} e_{13} e_{23} ; \phi_{012} \phi_{023}}\right)$. The complex tensor $C_{v_{0} v_{1} v_{2} v_{3} ; \phi_{013} e_{123}}^{e_{0} e_{02} e_{03} e_{12} e_{13} e_{23} \phi_{023}}$ can be associated with a tetrahedron which has a branching structure (see Fig. 5). A branching structure is a choice of orientation of each edge in the complex so that there is no oriented loop on any triangle (see Fig. 5). Here, the $v_{0}$ index is associated with the vertex 0 , the $e_{01}$ index is associated with the edge 01 , and the $\phi_{012}$ index is associated with the triangle 012 . They represent the degrees of freedom on the vertices, edges, and triangles. 


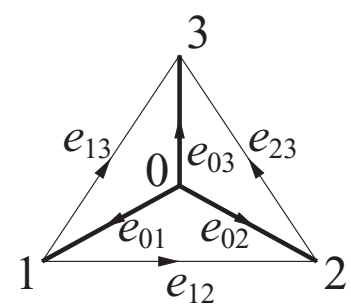

FIG. 5. The tensor $C_{v_{0} v_{1} v_{2} v_{3} ; \phi_{013} \phi_{123}}^{e_{01} e_{23} e_{3} e_{12} e_{13} e_{23} ; \phi_{012} \phi_{023}}$ is associated with a tetrahedron, which has a branching structure. If the vertex 0 is above the triangle 123 , then the tetrahedron will have an orientation $s_{0123}=*$. If the vertex 0 is below the triangle 123 , the tetrahedron will have an orientation $s_{0123}=1$. The branching structure gives the vertices a local order: the $i$ th vertex has $i$ incoming edges.

Using the tensors, we can define the topological path integral on any three-complex that has no boundary:

$$
\begin{aligned}
Z= & \sum_{v_{0}, \ldots ; e_{01}, \ldots ; \phi_{012}, \ldots} \prod_{\text {vertex }} w_{v_{0}} \prod_{\text {edge }} d_{e_{01}}^{v_{0} v_{1}} \\
& \times \prod_{\text {tetra }}\left[C_{v_{0} v_{1} v_{2} v_{3} ; \phi_{013} \phi_{123}}^{e_{01} e_{02} e_{03} e_{12} e_{13} e_{23} ; \phi_{012} \phi_{023}}\right]^{s_{0123}},
\end{aligned}
$$

where $\sum_{v_{0}, \ldots ; e_{01}, \ldots ; \phi_{012}, \ldots}$ sums over all the vertex indices, the edge indices, and the face indices and $s_{0123}=1$ or $*$ depending on the orientation of tetrahedron (see Fig. 5). We want to choose the tensors $\left(w_{v_{0}}, d_{e_{01}}^{v_{0} v_{1}}, C_{v_{0} v_{1} v_{2} v_{3} ; \phi_{013} \phi_{123}}^{e_{01} e_{02} e_{03} e_{12} e_{13} e_{23} ; \phi_{012} \phi_{023}}\right)$ such that the path integral is retriangulation invariant. Such a topological path integral describes a topologically ordered state in three space-time dimensions and also defines a topological order with a gappable boundary.

On the complex $C_{N}^{3}$ with boundary $B_{N^{\prime}}^{2}=\partial C_{N}^{3}$, the partition function is defined differently:

$$
\begin{aligned}
Z= & \sum_{\left\{v_{i} ; e_{i j} ; \phi_{i j k}\right\}} \prod_{\text {vertex } \notin B_{N^{\prime}}^{2}} w_{v_{0}} \prod_{\text {edge } \notin B_{N^{\prime}}^{2}} d_{e_{01}}^{v_{0} v_{1}} \\
& \times \prod_{\text {tetra }}\left[C_{v_{0} v_{1} v_{2} v_{3} ; \phi_{013} \phi_{123}}^{e_{01} e_{2} e_{03} e_{12} e_{13} e_{23} ; \phi_{012} \phi_{023}}\right]^{s_{0123}},
\end{aligned}
$$

where $\sum_{v_{i} ; e_{i j} ; \phi_{i j k}}$ sums over only the vertex indices, the edge indices, and the face indices that are not on the boundary. The resulting $Z$ is actually a complex function of $v_{i}$ 's, $e_{i j}$ 's, and $\phi_{i j k}$ 's on the boundary $B_{N^{\prime}}^{2}: Z=Z\left(\left\{v_{i} ; e_{i j} ; \phi_{i j k}\right\}\right)$. Such a function is a vector in $\mathcal{H}_{B_{N^{\prime}}^{2}}$. We will denote such a vector as $\left|\Psi\left(C_{N}^{3}\right)\right\rangle$.

We also note that the vertices and the edges are attached with the tensors $w_{v_{i}}$ and $d_{e_{01}}^{v_{0} v_{1}}$. But when we glue two boundaries together, those tensors $w_{v_{i}}$ and $d_{e_{i j}}^{v_{i} v_{j}}$ are added back. So the tensors $w_{v_{i}}$ and $d_{e_{i j}}^{v_{i} v_{j}}$ define the inner product in the boundary Hilbert space $\mathcal{H}_{B_{N^{\prime}}^{2}}$. Therefore, we require $w_{v_{i}}$ and $d_{e_{i j}}^{v_{i} v_{j}}$ to satisfy the following unitary condition:

$$
w_{v_{i}}>0, \quad d_{e_{i j}}^{v_{i} v_{j}}>0 .
$$

The invariance of $Z$ under the retriangulation in Fig. 6 requires that

$$
\begin{aligned}
& \sum_{\phi_{123}} C_{v_{0} v_{1} v_{2} v_{3} ; \phi_{013} \phi_{123}}^{e_{01} e_{02} e_{32} e_{12} e_{13} e_{23} ; \phi_{012} \phi_{023}} C_{v_{1} v_{2} v_{3} v_{4} ; \phi_{124} \phi_{234}}^{e_{12} e_{12} e_{14} e_{23} e_{24} e_{34} ; \phi_{123} \phi_{134}} \\
& =\sum_{e_{04}} d_{e_{04}}^{v_{0} v_{4}} \sum_{\phi_{014} \phi_{024} \phi_{034}} C_{v_{0} v_{1} v_{2} v_{4} ; \phi_{014} \phi_{124}}^{e_{01} e_{02} e_{04} e_{12} e_{14} e_{24} ; \phi_{012} \phi_{024}} C^{* e_{01} e_{03} e_{04} e_{13} e_{14} e_{34} ; \phi_{013} \phi_{034}} C_{v_{0} v_{1} v_{3} v_{4} ; \phi_{014} \nu_{3} v_{4} ; \phi_{024} \phi_{234}}^{e_{02} e_{03} e_{04} e_{23} e_{24} e_{34} ; \phi_{023} \phi_{034}}
\end{aligned}
$$

We would like to mention that there are other similar conditions for different choices of the branching structures. The branching structure of a tetrahedron affects the labeling of the vertices.

The invariance of $Z$ under the retriangulation in Fig. 7 requires that

$$
\begin{aligned}
& C_{v_{0} v_{2} v_{3} v_{4} ; \phi_{024} \phi_{234}}^{e_{02} e_{03} e_{04} e_{23} e_{24} e_{34} ; \phi_{023} \phi_{034}}=\sum_{e_{01} e_{12} e_{13} e_{14}, v_{1}} w_{v_{1}} d_{e_{01}}^{v_{0} v_{1}} d_{e_{12}}^{v_{1} v_{2}} d_{e_{13}}^{v_{1} v_{3}} d_{e_{14}}^{v_{1} v_{4}} \sum_{\phi_{012} \phi_{013} \phi_{014} \phi_{123} \phi_{124} \phi_{134}} \\
& \times C_{v_{0} v_{1} v_{2} v_{3} ; \phi_{013} \phi_{123}}^{e_{01} e_{02} e_{03} e_{12} e_{13} e_{23} ; \phi_{012}} \phi_{023} C^{* e_{01} e_{02} e_{04} e_{12} e_{14} e_{24} ; \phi_{012} \phi_{024}} C_{v_{0} v_{1} v_{2} v_{4} ; \phi_{014} \phi_{124}}^{e_{01} e_{03} e_{04} e_{13} e_{14} e_{34} ; \phi_{013} \phi_{034}} C_{v_{0} v_{1} v_{3} v_{4} ; \phi_{014} \phi_{134}}^{e_{12} e_{13} e_{14} e_{23} e_{24} e_{34} ; \phi_{123} \phi_{134}}
\end{aligned}
$$

Again, there are other similar conditions for different choices of the branching structures.

The above two types of conditions are sufficient for producing a topologically invariant partition function $Z$, which is nothing but the topological invariant for three manifolds introduced by Turaev and Viro [32]. Again, two different solutions are regarded as equivalent if they produce the same topology-dependent partition function for any closed spacetime.

It is also clear that the above construction of topological path integrals can be easily generalized to any other dimension. This gives rise to a classification of topological orders with a gappable boundary in higher dimensions.

\section{APPENDIX C: QUANTUM VOLUME AND ITS PROPERTIES}

We have seen that when a tensor set, for example, $T=$ $\left(w_{v_{0}}, d_{e_{01}}^{v_{0} v_{1}}, C_{v_{0} v_{1} v_{2} v_{3} ; \phi_{013} e_{123}}^{e_{01} e_{02} e_{03} e_{2} e_{13} e_{23} ; \phi_{012} \phi_{023}}\right)$, satisfies conditions (B6)

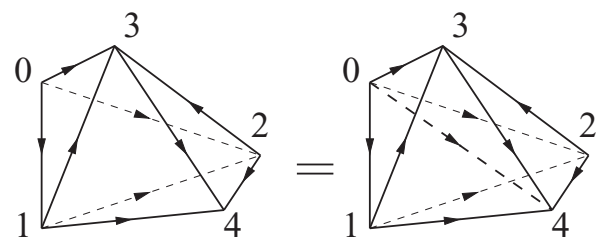

FIG. 6. A retriangulation of a 3D complex. 


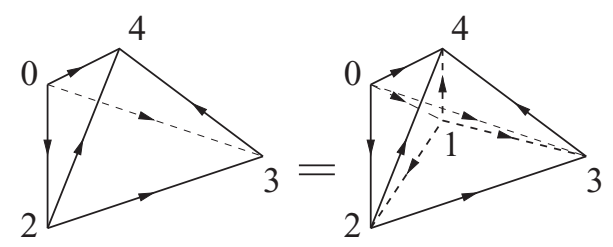

FIG. 7. A retriangulation of another $3 \mathrm{D}$ complex.

and (B7), its path integral on different space-time complexes will describe the same topological phase. If we change the tensors by a small amount, the tensor set will still describe the same topological phase for different space-time complexes. With such a more careful definition of path integral in terms of the tensor set and the space-time complex $C_{N}^{d+1}$ with the branching structure, we can define quantum volume more precisely.

For example, on $(2+1) \mathrm{D}$ space-time complex $C_{N}^{3}$ with boundary $B_{N^{\prime}}^{2}$, the path integral produces a complex function $Z\left(\left\{v_{i} ; e_{i j} ; \phi_{i j k}\right\}\right)$, with $\left\{v_{i} ; e_{i j} ; \phi_{i j k}\right\} \subset B_{N^{\prime}}^{2}$, which is a vector $\left|\Psi\left(C_{N}^{3}\right)\right\rangle \in \mathcal{H}_{B_{N^{\prime}}^{2}}$ [see Eq. (B4)]. The inner product in $\mathcal{H}_{B_{N^{\prime}}^{2}}$ is defined through the weight tensors $w_{v_{i}}$ and $d_{e_{i j}}^{v_{i} v_{j}}$ :

$$
\langle\Psi \mid \Psi\rangle=\sum_{\left\{v_{i} ; e_{i j} ; \phi_{i j k}\right\}} \prod_{i} w_{v_{i}} \prod_{\langle i j\rangle} d_{e_{i j}}^{v_{i} v_{j}}\left|Z\left(\left\{v_{i} ; e_{i j} ; \phi_{i j k}\right\}\right)\right|^{2} .
$$

In this case the classical volume of $C_{N}^{3}$ is given by

$$
V\left(C_{N}^{3}, T\right)=\ln \sqrt{\left\langle\Psi\left(C_{N}^{3}, T\right) \mid \Psi\left(C_{N}^{3}, T\right)\right\rangle} .
$$

We can show that, for a many-body system described by tensor set $T$, its q-volume satisfies

$$
\begin{aligned}
& \left|\Psi\left(C_{1}^{d+1} \cup C_{2}^{d+1}\right)\right\rangle \\
& \quad=\sum_{\partial C_{1}^{d+1} \cap \partial C_{2}^{d+1}}\left[\left|\Psi\left(C_{1}^{d+1}\right)\right\rangle \otimes\left|\Psi\left(C_{2}^{d+1}\right)\right\rangle\right]
\end{aligned}
$$

if the space-time complexes $C_{1}^{d+1}$ and $C_{2}^{d+1}$ overlap only on their boundaries. We note that $\left|\Psi\left(C_{1}^{d+1}\right)\right\rangle$ and $\left|\Psi\left(C_{2}^{d+1}\right)\right\rangle$ are complex functions that depend on the indices on the boundaries $\partial C_{1}^{d+1}$ and $\partial C_{2}^{d+1}$. Here, $\sum_{\partial C_{1}^{d+1} \cap \partial C_{2}^{d+1}}$ sums over the internal degrees of freedom on the overlapped boundaries $\partial C_{1}^{d+1} \cap \partial C_{2}^{d+1}$ with the weighting tensors $w_{v_{1}}, d_{e_{12}}^{v_{1} v_{2}}$, etc., for the internal simplices on the overlapped boundaries. In other words, we trace over only the indices for the simplices inside the overlapped boundaries (not those for the simplices on the boundary of the overlapped boundaries). The summation of each index is weighted by the corresponding weight tensor $w_{v_{1}}$ or $d_{e_{12}}^{v_{1} v_{2}}$ and so on. Equation (C3) is a key property of the quantum volume.
[1] X.-G. Wen, Phys. Rev. D 68, 065003 (2003).

[2] M. Levin and X.-G. Wen, Phys. Rev. B 73, 035122 (2006).

[3] X.-G. Wen, Chin. Phys. Lett. 30, 111101 (2013).

[4] Y.-Z. You, Y. BenTov, and C. Xu, arXiv:1402.4151.

[5] Y.-Z. You and C. Xu, Phys. Rev. B 91, 125147 (2015).

[6] J. Maldacena, Adv. Theor. Math. Phys. 2, 231 (1998).

[7] S.-S. Lee, Nucl. Phys. B 832, 567 (2010).

[8] C. Xu, arXiv:cond-mat/0602443.

[9] Z.-C. Gu and X.-G. Wen, arXiv:gr-qc/0606100.

[10] Z.-C. Gu and X.-G. Wen, Nucl. Phys. B 863, 90 (2012).

[11] E. Witten, Commun. Math. Phys. 121, 351 (1989).

[12] M. Atiyah, Publ. Math. IHES 68, 175 (1988).

[13] X. G. Wen, Int. J. Mod. Phys. B 04, 239 (1990).

[14] X. G. Wen and Q. Niu, Phys. Rev. B 41, 9377 (1990).

[15] B. Zeng and X.-G. Wen, Phys. Rev. B 91, 125121 (2015).

[16] B. Swingle and J. McGreevy, Phys. Rev. B 93, 045127 (2016).

[17] C. Chamon, Phys. Rev. Lett. 94, 040402 (2005).

[18] J. Haah, Phys. Rev. A 83, 042330 (2011).

[19] W. Shirley, K. Slagle, Z. Wang, and X. Chen, Phys. Rev. X 8, 031051 (2018).

[20] W. Shirley, K. Slagle, and X. Chen, SciPost Phys. 6, 015 (2019).

[21] A. Kitaev and J. Preskill, Phys. Rev. Lett. 96, 110404 (2006).
[22] M. Levin and X.-G. Wen, Phys. Rev. Lett. 96, 110405 (2006).

[23] L. Kong and X.-G. Wen, arXiv:1405.5858.

[24] M. Gromov, mathématiques de l'I.H.É.S. 56, 5 (1982).

[25] Z.-C. Gu and X.-G. Wen, Phys. Rev. B 80, 155131 (2009).

[26] J. Wang, X.-G. Wen, and S.-T. Yau, Ann. Phys. (N.Y.) 409, 167904 (2019).

[27] E. Keski-Vakkuri and X.-G. Wen, Int. J. Mod. Phys. B 7, 4227 (1993).

[28] Y. Zhang, T. Grover, A. Turner, M. Oshikawa, and A. Vishwanath, Phys. Rev. B 85, 235151 (2012).

[29] F. Costantino, Math. Z. 251, 427 (2005).

[30] X. Chen, Z.-C. Gu, Z.-X. Liu, and X.-G. Wen, Phys. Rev. B 87, 155114 (2013).

[31] X. Chen, Z.-C. Gu, Z.-X. Liu, and X.-G. Wen, Science 338, 1604 (2012).

[32] V. Turaev and O. Viro, Topology 31, 865 (1992).

[33] M. A. Levin and X.-G. Wen, Phys. Rev. B 71, 045110 (2005).

[34] A. Kirillov, Jr., arXiv:1106.6033.

[35] B. Balsam and A. Kirillov, Jr., arXiv:1206.2308.

[36] X. Chen, Z.-C. Gu, and X.-G. Wen, Phys. Rev. B 82, 155138 (2010).

[37] F. Verstraete and J. I. Cirac, arXiv:cond-mat/0407066.

[38] M. Levin and C. P. Nave, Phys. Rev. Lett. 99, 120601 (2007). 